probably owing to decomposition or ring-formation. The substances melt with simultaneous decomposition, already showing some browning below the decomposition point.

$\begin{array}{lll} & {[a]_{D}^{\prime \prime}} & \text { Decomp. point } \\ \text { Bovine sialic acid } & +8^{\circ} \pm 2^{\circ} & 152-153^{\circ} \text { (uncorr.) } \\ \text { Porcine ", ", } & \pm 31^{\circ} \pm 2^{\circ} & 185-187^{\circ} \\ \text { Ovine " " } & -32^{\circ} \pm 2^{\circ} & 185-187^{\circ} \quad,\end{array}$

The 2-carboxy-pyrrole found by Gottschalk ${ }^{5}$ as a product of splitting on alkaline hydrolysis of submaxillary mucin is no doubt formed by a cyclization process during the alkaline decomposition of sialic acid. The neuraminic acid of Klenk ${ }^{6}$, the hæmataminic acid of Yamakawa and Suzuki ${ }^{7}$, and the lactaminic acid of $\mathrm{Kuhn}^{8}$ obviously all bear a very close relation to sialic acid. The first two probably simply represent de-acetylated sialic acids.

A detailed report of this work will appear elsewhere.

Note added in proof. The assumption of the presence of a branched carbon chain in the sialic acid molecule has received additional support by recent micro hydroxyl-group determinations. These revealed six hydroxyl groups per molecule in the porcine sialic acid and five in that of the bovine substance.

Institute of Medical Chemistry,
G. BLIX
E. LindBiera
L. ODIN
I. WERNER

University of Uppsala. Oct. 27.

2 Blix, G., Z. physiol. Chem., 240, 43 (1936). Blix, G., Svennerholm, L., and Werner, I., Acta Chem. Scand.,
6, 358 (1952).

'Werner, T., and Odin, L., Acta Med. Soc. Upsal., 57, 230 (1952).

4 Tobie, W. C. Indust Eng. Chem., 14, 405 (1942).

'Gottschalk, A., Nature, 174, 652 (1954).

- Klenk, E., Z. physiol. Chem., 268, 50 (1941).

- Yamakawa, T., and Suzuki, S., J. Biochem., Japan, 38, 199 (1951).

- Kuhn, R., and Brossmer, R., Ber. deut. chem. Ges., 87, 123 (1954).

\section{Biological Decomposition of Aromatic Nitro-compounds}

Certain organic nitro-compounds, for example, chloramphenicol, are known to arise biologically; but very little is known about the transformation of this class of compounds by micro-organisms. Erikson ${ }^{1}$ and Moore $^{2}$ found that certain Actinomycetes could utilize picric acid, trinitroresorcinol, and nitrobenzene, and Simpson and Evans ${ }^{3}$ have reported briefly on species of Pseudomonas that decompose 0 - and $p$-nitrophenol with the formation of nitrite; the last workers also mention nitrite production from 2,4-dinitrophenol and chloramphenicol.

According to a personal communication from Mr. H. Ingvard Petersen (Government Weed Research Department, Lyngby, Denmark), the herbicide dinitro-ortho-cresol is rapidly inactivated in soil, apparently by biological agencies, whereas the chemically related herbicide dinitrobutylphenol ap. pears more persistent. From the soil used in these detoxication experiments, we have isolated an organism that decomposes 4,6-dinitro-ortho-cresol and several related compounds with release of nitrite.

The organism appears in young cultures as a nonspore-forming, Gram-positive, irregular rod, approximately 1.4-4.0 $\mu \times 0 \cdot 6-0 \cdot 8 \mu$, in characteristic angular or parallel arrangements ; the cells in older cultures become shorter or almost coccoid, $0 \cdot 7-1 \cdot 3 \mu$ in

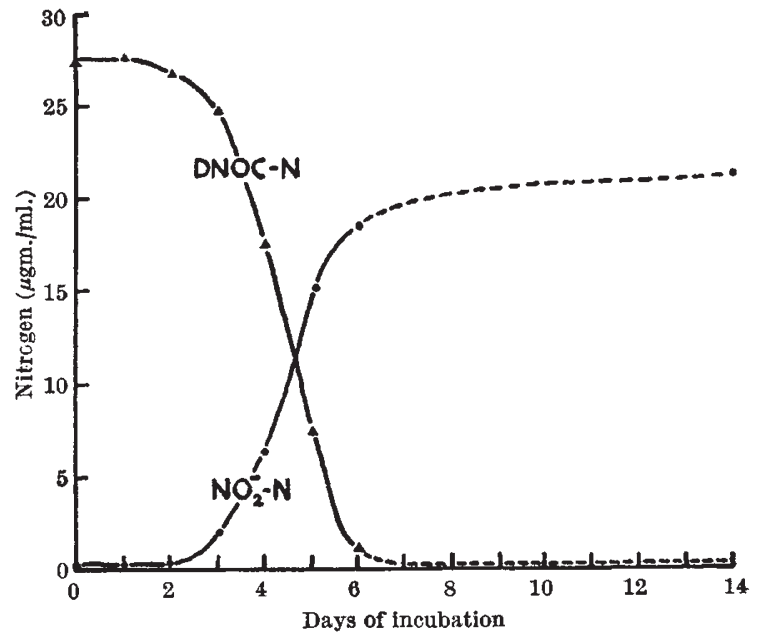

Fig. 1. Production of nitrite from dinitro-ortho-cresol (approx. $1^{-} ; M$ ) by Corynebacterium sp. (simplex ?) in liquid medium incubated at $25^{\circ} \mathrm{C}$.

diameter. Taxonomically, it seems to be an atypical form of Corynebacterium simplex ${ }^{4}$. Decomposition of dinitro-ortho-cresol in solution as well as in agar medium is evidenced by the bleaching of the yellow colour and the appearance of nitrite. Growth is possible in a synthetic medium with no other organic constituents than $0.01-0.05$ per cent dinitro-orthocresol (as sodium salt), but is slow and unreliable under these conditions; addition of 0.01-0.1 per cent yeast extract greatly accelerates growth and nitrite formation. The results of an experiment in liquid medium with 0.02 per cent dinitro-ortho-cresol and 0.01 per cent yeast extract are shown in Fig. 1 . (Nitrite and residual dinitro-ortho-cresol were both determined colorimetrically on a Coleman spectrophotometer, the former by means of the Gries reagent, the latter directly by the intensity of the yellow colour.)

Besides 4,6-dinitro-ortho-cresol, the organism decomposes $p$-nitrophenol, 2,4-dinitrophenol, and 2,4,6trinitrophenol (picric acid), but not $o$ - and $m$-nitrophenol, 2,5- and 2,6-dinitrophenol or 3,5-dinitrobenzoic acid. Thus only compounds with a nitrogroup in the para-position appear liable to attack; we have not, however, been able to detect nitrite formation from 4,6-dinitrobutylphenol, which also seems to be less readily inactivated in soil, as mentioned above. The results in Fig. 1, as well as similar experiments with dinitrophenol, show that more than half the nitrogen in dinitro-compounds is converted into nitrite, that is, both nitro-groups are metabolized. Possibly the $p$-group represents the point where the enzymatic attack begins and the ring-structure is broken, with subsequent release of the second nitro-group.

The results of these experiments will be published in detail elsewhere.

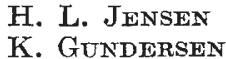

State Laboratory for Plant Culture,

Department of Bacteriology, Lyngby, Denmark. Oct. 25.

${ }^{2}$ Erikson, D., J. Bact., 41, 277 (1941).

${ }^{2}$ Moore, F. W., J. Gen. Microbiol., 3, 143 (1949).

${ }^{3}$ Simpson, J. R., and Evans, W. C., Biochem. J.. 55, (2), xxiv (1953).

"Bergey, D. H. "Manual of Determinative Bacteriology" (Baillière, Tindall and Cox, London, 6th edit., 1948). 